\title{
Propagating structures in drift-wave turbulence
}

\author{
T. Windisch*, O. Grulke and T. Klinger \\ Max-Planck-Institute for Plasma Physics, EURATOM Association, Greifswald, Germany
}

\begin{abstract}
The formation and propagation of spatiotemporal fluctuation structures in drift-wave turbulence in a linearly magnetized helicon device is investigated. Structures are extracted by correlation analysis. Their typical lifetime exceeds the eddy turnover time by one order of magnitude. The radial correlation analysis yields radial propagation of turbulent fluctuations with velocities up to $50 \%$ of the ion sound speed. The two-dimensional analysis of the entire azimuthal cross-sections reveals that the propagation is purely azimuthal and that the radial speeds are an artefact of the one-dimensional measurement of a multi-dimensional propagation.
\end{abstract}

PACS numbers: 52.35.Ra,52.35.Kt

\section{INTRODUCTION}

Motivated by the observation of particle and energy losses perpendicular to the magnetic field in fusion devices, the fluctuation induced cross-field transport has been deeply investigated over the last two decades. It was found in tokamak experiments [1-3] and in numerical simulations [4] that radially propagating spatiotemporal structures, so-called blobs, contribute considerably to the cross-field transport. Recently, in Ref. [5] some indication has been reported that blobs also develop in devices with linear magnetic geometry. In this paper we characterize the propagation of turbulent structures in driftwave turbulence in the linear VINETA device. Crosscorrelation techniques are used as a statistical tool to reconstruct the spatiotemporal dynamics of the fluctuations. The paper is organized as follows: The experimental setup is described in section II. Section III deals with the basic statistical fluctuation properties. The propagation of spatiotemporal structures is characterized in section IV.

\section{EXPERIMENTAL SETUP AND STATISTICAL METHODS}

The experiments were carried out in the linearly magnetized helicon device VINETA [6]. It consists of four separate stainless steel vessels with a diameter of $0.4 \mathrm{~m}$ and has total length of $4.5 \mathrm{~m}$ (Fig. 1).

The vacuum vessel is immersed in a set of 36 magnetic field coils which provide a homogeneous axial magnetic field up to 0.1 T. For plasma production we use a conventional helicon source [7]. The source consists of a helical $m=+1$ antenna, which is placed around a cylindrical glass vacuum extension of $0.1 \mathrm{~m}$ diameter and $0.5 \mathrm{~m}$ length. The antenna is coupled to a rf source $(f=13.56$ $\mathrm{MHz}, P \leq 5 \mathrm{~kW})$ via a capacitive matching unit. For the described experiments the Argon gas pressure is 0.04

\footnotetext{
*electronic mail: thomas.windisch@ipp.mpg.de
}

TABLE I: Typical plasma parameters.

\begin{tabular}{lr}
\hline \hline Plasma parameter & Typical value \\
\hline Peak electron density $n$ & $1 \cdot 10^{19} \mathrm{~m}^{-3}$ \\
Electron temperature $T_{e}$ & $2.5 \mathrm{eV}$ \\
Ion temperature $T_{i}$ & $0.2 \mathrm{eV}$ \\
Ion gyro frequency $\omega_{c i}$ & $2.5 \cdot 10^{5} \mathrm{rad} / \mathrm{s}$ \\
Ion gyro radius $r_{c i}$ & $4 \mathrm{~mm}$ \\
Drift-scale $\rho_{s}$ & $1.4 \mathrm{~cm}$ \\
Peak plasma $\beta$ & $10^{-3}$ \\
\hline \hline
\end{tabular}

$\mathrm{Pa}$ and the rf power is $1.8 \mathrm{~kW}$. As a general feature of helicon sources, a rather dense plasma with low electron temperatures is produced. Typical plasma parameters are compiled in table I.

The Argon helicon plasma generated in the VINETA device is characterized by fairly high peak plasma $\beta$ $\approx 1 \%$ and high collisionality $\omega_{c i} / \nu_{e i} \approx 5 \cdot 10^{-4}$. Time averaged plasma parameter profiles are measured with rfcompensated Langmuir probes [8]. Plasma fluctuations are measured with uncompensated Langmuir probes. Since temperature fluctuations are negligible, we can approximate fluctuations of plasma density by fluctuations of the ion saturation current and fluctuations of plasma potential by fluctuations of the floating potential. To gain insight in the azimuthal dynamics we use an azimuthal $x y$ - probe array to measure the fluctuating quantities simultaneously at 56 positions in the azimuthal plane. The 56 single probes have a spatial spacing of $1.7 \mathrm{~cm}$. A schematic diagram and the localization of the probe array with respect to the plasma density contour is shown in Fig. 2.

Time series of plasma density or plasma potential fluctuations are recorded with high temporal resolution (1.25 $\mathrm{MHz}$ sampling frequency), 12 bit amplitude resolution and a record length of $65 \mathrm{k}$ samples. Turbulent structures are extracted by calculating the two-point crosscorrelation function $C(d, t)=<f(\mathbf{x}, \mathrm{t}) \mathrm{g}(\mathbf{x}+\mathbf{d}, \mathrm{t}+\tau)>$, which determines the phase coherence between two time series. A single probe acts as a reference probe, located at $\Delta z=0.43 \mathrm{~m}$ upstream from the probe array at a radial position of maximum density gradient and thus maximum fluctuation degree $(r=35 \mathrm{~mm})$. The cross- 


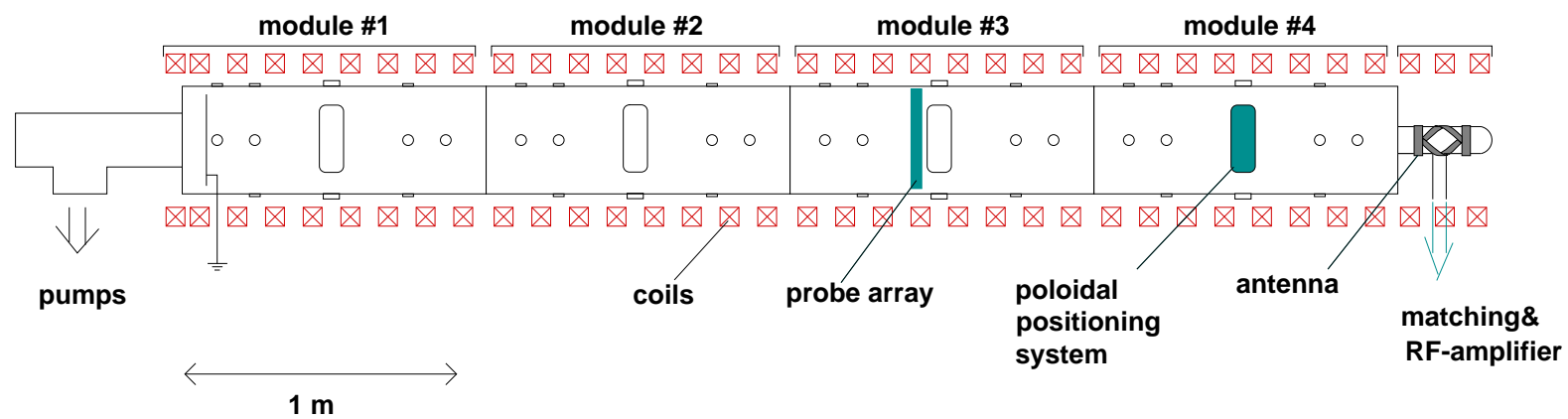

FIG. 1: Schematics of the VINETA device.

correlation function of plasma density and plasma potential fluctuations recorded with the reference probe $f(\mathbf{x}, \mathrm{t})$ and the probe array $g(\mathbf{x}+\mathbf{d}, \mathrm{t}+\tau)$ then yields the reconstruction of the azimuthal dynamics in space and time.

\section{BASIC CHARACTERISTICS OF ELECTROSTATIC FLUCTUATIONS}

The governing instability in the VINETA device is the drift-wave instability [9]. Drift-waves are low-frequency waves $\left(\omega<<\omega_{c i}\right)$ in magnetized plasmas, that are driven by the free energy provided by pressure gradients perpendicular to the ambient magnetic field. For a review on drift-waves and related plasma transport, the reader is referred to Ref. [11]. As shown in Ref. [9], single coherent drift-wave modes with frequencies $\omega / 2 \pi \approx \omega^{\star} / 2 \pi=1-10 \mathrm{kHz}$ ( $\omega^{\star}$ being the electron diamagnetic drift frequency) and poloidal mode numbers $m=1-8$ can be excited in the VINETA device. In the case of single saturated drift-wave modes, the phase shift between density and potential fluctuations is observed to

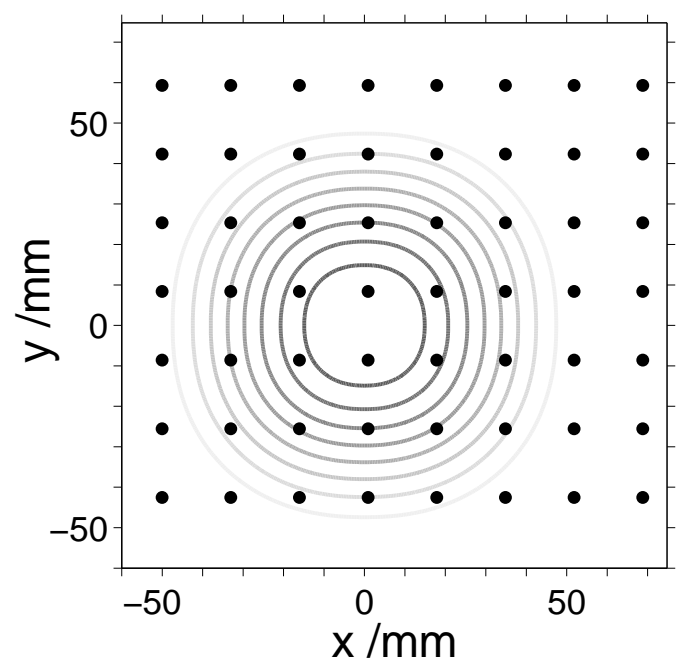

FIG. 2: The probe array and its position relative to the plasma density profile. be $\angle(\tilde{n}, \tilde{\phi})<45^{\circ}$ and the parallel wavelength is mainly determined by the machine length [9].
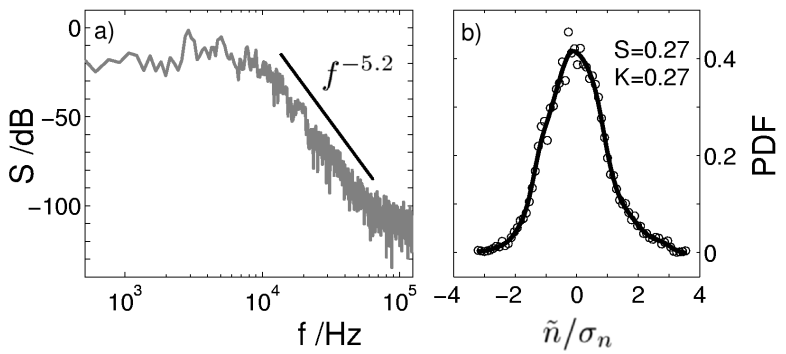

FIG. 3: Power spectrum of density fluctuations (a) and their probability density function (PDF) with its higher order moments (b).

Since the plasma is weakly ionized ( $10 \%$ ionization degree in the maximum density gradient region), the major destabilizing effect are electron-neutral collisions [10]. The external control parameter for the destabilization of different drift wave modes is the ambient magnetic field.

The turbulent state of the plasma is characterized by a continuous frequency spectrum. The power spectrum of density fluctuations measured with a single probe of the probe array is shown in Fig. 3a. The power spectrum has one scaling only region which can be described by a power law $S(f) \propto f^{-\alpha}$ with $\alpha=-5.2$, as expected for driftwave turbulence $[12,13]$. The smaller peaks in the inertial range of the power spectrum indicate that the turbulent state is not yet fully developed. The corresponding probability density function (PDF) is single-humped and is close to a Gaussian distribution. This is confirmed by the moments of the PDF, i.e. the skewness $s=0.27$ is relatively small and the kurtosis $k=0.27$ indicates a slight peaking compared to a Gaussian distribution. Generally speaking, skewness and kurtosis greatly increase for radial probe positions in the residual plasma $(r>35 \mathrm{~mm}$ far at the edge), indicating large positive density bursts. The rms-value of density fluctuations reaches $20 \%$ at the position of maximum radial density gradient. 


\section{PROPAGATION OF FLUCTUATION STRUCTURES}

A straightforward measure of the radial propagation of fluctuation structures is done by correlation analysis along the radial direction. In our experiment, the radial velocity of density fluctuations is measured by using 6 probe tips in $x$-direction, centered in $y$-direction of the probe array. One of these probes acts as a reference probe and the cross-correlation function of this reference signal and the other probe signals is calculated as usual.
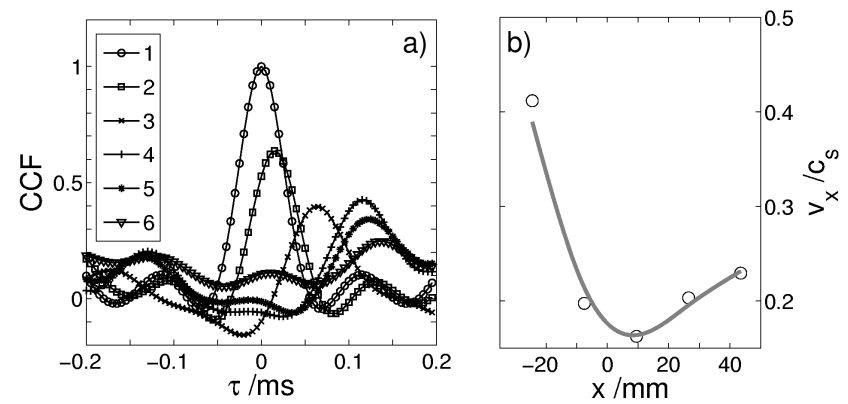

FIG. 4: Radial cross-correlation function of density fluctuations for the centered 6 probe tips (a) and the calculated radial velocity (b).

The resulting cross-correlation amplitude for density fluctuations of the single probes is shown in Fig. 4a. The first two probes are located on the $-x$-axis with respect to the plasma center and the other four probes are located on the $+x$-axis, such that the entire radial plasma density profile is covered. Fig. 4a shows that the correlation-function for an increasing probe number achieves a maximum value at increasing time lags. This actually indicates radial propagation. Based on the time shift of the maximum correlation and the probe spacing, the corresponding radial velocities are calculated and are shown in Fig. 4b. The radial velocity peaks asymmetrically at the edge of the plasma density profile with a maximum velocity of $0.4 \cdot c_{s}\left(c_{s} \approx 2800 \mathrm{~m} / \mathrm{s}\right.$ is the ion sound speed) and decreases towards the plasma center. Note that the cross-correlation amplitude in Fig. 4a decreases for the first four probe tips, as expected, but increases for probe tip five, which is located at the same radial position as probe tip one but on the $+x$-axis with respect to the center.

To gain more insight into the propagation details, the spatial fluctuation structure in the azimuthal crosssection is considered. The cross-correlation of density fluctuations between the reference probe and the probe array is presented in Fig. 5 for a time lag $\tau=-8 \mu \mathrm{s}$. For comparison the cross-correlation between potential and density fluctuations is also included in Fig. 5 as contour lines. In both cases a large azimuthally elongated monopole-like structure is observed. The phase shift between density and potential fluctuation is found to be small $\left(\approx 10^{\circ}\right)$, consistent with previous findings for sin-

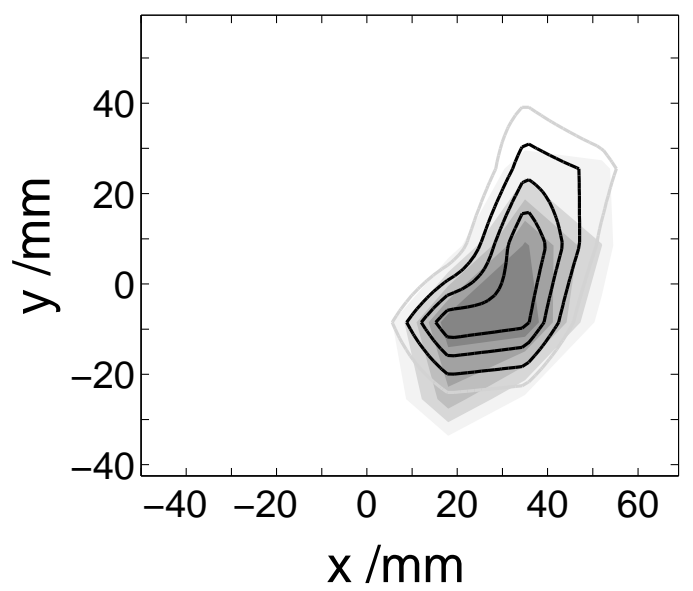

FIG. 5: Comparison between density and potential fluctuations. Density-density (filled contour) and potential-density (contour lines) cross-correlation between the reference probe and the probe array.

gle coherent drift wave modes. The associated electric field at the turbulent structure yields an eddy turnover time $\approx 10 \mu \mathrm{s}$. The propagation of the structure is reconstructed in Fig. 6, which shows the cross-correlation of density fluctuations between the reference probe and the probe array for three different time lags. The structure forms, propagates and decays with a correlation time $\tau_{c}=159 \mu \mathrm{s}$, which is much longer than the eddy turnover time. This is a characteristic feature of coherent structures [14]. The correlation length $l_{c} \approx 2 \mathrm{~cm}$ is comparable to the scale size of saturated drift-modes. As the black ring in Fig. 6 indicates, the propagation direction is purely azimuthal in the direction of the electron diamagnetic drift direction (which is anti-parallel to the background $\mathrm{E} \times \mathrm{B}$-drift) with an azimuthal velocity of 760 $\mathrm{m} / \mathrm{s}$.

\section{SUMMARY}

It is investigated the propagation of a large coherent structure, which forms in drift-wave turbulence. The turbulent state of the plasma is affirmed by the spectral properties of density fluctuations. The power spectral density shows a power law scaling with a spectral index of 5.2. The PDF of density fluctuations is close to a Gaussian distribution and slightly skewed towards positive values. Radial correlation analysis yields radial velocities of fluctuating structures up to $40 \%$ of $c_{s}$. This radial velocity cannot be explained by any error due to a misalignment of the probe array relative to the plasma center. Correlation analysis in the entire azimuthal cross-section shows that, indeed, high correlation amplitudes can be ascribed to spatiotemporal fluctuations structures. The phase $\left(\approx 10^{\circ}\right)$ between density and potential structures is small and reflects the features of drift-wave modes. The typical eddy turnover time is one 

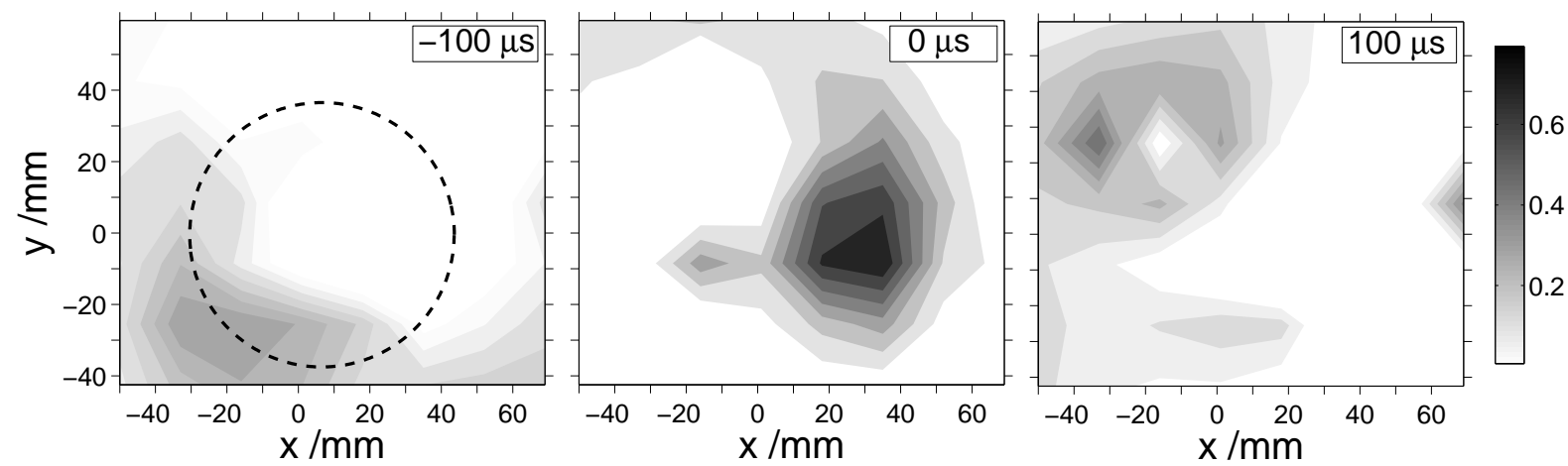

FIG. 6: Density cross-correlation between the reference probe and the probe array for three different time lags. The black ring indicates the radial position of maximum density gradient $\left(r_{p}=35 \mathrm{~mm}\right)$.

order of magnitude smaller than the structures' lifetime. Thus, one deals with coherent structures in the strict sense, consistent with previous measurements of structures in drift-wave turbulence [14]. Their propagation is found to be purely in azimuthal direction. The azimuthal propagation causes the observed increase of the crosscorrelation amplitude of probe tip 5 in Fig. 4a. Thus the azimuthal velocity calculated from the one-dimensional measurement $(800 \mathrm{~m} / \mathrm{s})$ agrees with the former result of
$760 \mathrm{~m} / \mathrm{s}$. For comparison, the electron diamagnetic drift velocity and the $\mathrm{E} \times \mathrm{B}$ velocity at the same radial position equal $1300 \mathrm{~m} / \mathrm{s}$ and $2600 \mathrm{~m} / \mathrm{s}$ respectively. To conclude, our finding of a purely azimuthally propagation of the coherent structure stands in contrast to the radial correlation analysis and shows that the one-dimensional analysis is not sufficient to characterize the multi-dimensional dynamics of structures.
[1] J. L. Terry et al, Phys. Plasm. 10, 1739 (2003).

[2] J. A. Boedo et al, Phys. Plasm. 10, 1670 (2003).

[3] S. J. Zweben et al, Nucl. Fus. 44, 134 (2004).

[4] O. E. Garcia, V. Naulin, A. H. Nielsen and J. J. Rasmussen, Phys. Rev. Lett. 92 165003, (2004).

[5] G. Y. Antar et al, Phys. Plasm. 10, 419 (2003).

[6] C. M. Franck, O. Grulke and T. Klinger, Phys. Plasm. 10, 323 (2003).

7] R. W. Boswell, Plas. Phys. Contr. Fus. 26, 1147 (1984).

[8] I. D. Sudit and F. F. Chen, Plasma Sources Sci. Technol. 3, 162 (1994).
[9] C. Schroeder, O. Grulke, T. Klinger and V. Naulin, Phys. Plasm. 12, 042103 (2005).

[10] C. Schroeder, O. Grulke and T. Klinger, Phys. Plasm. 11, 4249 (2004).

[11] W. Horton, Rev. Mod. Phys. 71, 735 (1999).

[12] M. C. Kelley, Phys. Fluids 25, 1002 (1982).

[13] H. L. Pécseli, Physica Scripta A T2, 147 (1982).

[14] O. Grulke, T. Klinger and A. Piel, Phys. Plasm. 6, 788 (1999). 